\title{
Mathematische Optimierung in der Schule
}

\author{
Alexander Schulte
}

\begin{abstract}
Lineare und Ganzzahlige Programmierung als Teil der mathematischen Optimierung sind ein wichtiges Teilgebiet des Operations Research und damit der angewandten Mathematik. Das vorgestellte Unterrichtsbeispiel, konzipiert für die Jahrgangsstufe 9/10, führt anhand des bekannten Sudoku-Rätsels in dieses Thema ein. Ein Schwerpunkt liegt dabei auf der Vermittlung von authentischen wissenschaftlichen Arbeits- und Denkweisen. Die Einführung in die (ganzzahlige) mathematische Modellbildung erfolgt durch die Entwicklung eines ganzzahligen Modells von Sudoku sowie die Vorstellung gängiger Bestandteile von Baumsuchalgorithmen (Propagierung, Probing). Durch die authentische Herangehensweise findet ein produktiver Umgang mit dem Thema statt, der zu einem tiefen Verständnis für die elementaren Prinzipien der mathematischen Optimierung führt.
\end{abstract}

Das Schulfach Mathematik bestimmt für viele Schülerinnen und Schüler nachhaltig das eigene Bild von Mathematik. Die behandelten mathematischen Inhalte haben sich bis auf wenige Ausnahmen (beispielsweise Mengenlehre und Wahrscheinlichkeitsrechnung) in den letzten 1 oo Jahren kaum verändert. So verwundert es kaum, dass in der breiten Öffentlichkeit oftmals Mathematik mit Rechnen gleichgesetzt wird und es häufig an konkreten Vorstellungen fehlt, was das Forschungs- und Arbeitsfeld insbesondere der angewandten Mathematik ausmacht.

Im aktuellen Berliner Rahmenlehrplan für das Fach Mathematik wird als eines der Ziele des Mathematikunterrichts genannt:

Die Schülerinnen und Schüler erkennen Mathematik mit ihrer fachspezifischen Sprache, ihren Symbolen, Bildern, Darstellungen und Formeln als ein eigenes, geordnetes Konzept und nutzen sie, um mathematische Aufgaben zu beschreiben und zu bearbeiten [3].

Damit werde die Grundlage für die lebenslange Auseinandersetzung mit mathematischen Anforderungen des täglichen Lebens und der Berufswelt gelegt, sowie Anknüpfungspunkte für weiteres, nachhaltiges Lernen im Fach Mathematik geschaffen.

Mathematik wird in der Schule also als Denkdomäne wahrgenommen, die das logische und strukturierte Urteilen fördert. Gleichzeitig werden der Anwendungsaspekt von Mathematik betont und der Werkzeugcharakter herausgestellt. Allerdings kommen die Lernenden in der Schule in der Regel kaum mit moderner angewandter Mathematik in Berührung. So taucht beispielsweise Lineare und Ganzzahlige Programmierung als eine aus der Mathematik stammende Schlüsseltechnologie des 20. Jahrhunderts im Bildungskanon bis auf wenige Ausnahmen kaum auf. Die Auseinandersetzung mit diesem Thema bietet sich jedoch in besonderem Maße an, um Schülerinnen und Schülern einerseits mit modernen Methoden der angewandten Mathematik zu konfrontieren und andererseits mathematische Denkstrukturen zu erweitern.
1 Ganzzahlige Programmierung in der Schule am Beispiel von Sudoku

Das im Folgenden vorgestellte Unterrichtsvorhaben führt anhand des bekannten Sudoku-Rätsels in die mathematische Programmierung ein. Die Unterrichtseinheit wurde in einem Wahlpflichtkurs Mathematik (9. Schuljahr) an einem Berliner Gymnasium durchgeführt. Der Zugang über Sudoku bietet sich insofern an, da die meisten Schülerinnen und Schüler schon mit diesem Rätsel in Berührung gekommen sind. Die Regeln und die gängigen „händischen“ Lösungsstrategien des Sudoku-Rätsels sind mehrheitlich bekannt. Außerdem bietet das Rätsel durch die einfache Struktur seiner Regeln einen intuitiven Zugang zur mathematischen Modellbildung (vgl. [2]).

\subsection{Ein ganzzahliges Modell von Sudoku}

In einer ersten Auseinandersetzung entwickelten die Lernenden eigene Ideen, das Sudoku mit seinen Regeln in Mathematik zu übersetzen. Zunächst wurde das klassische $9 \times 9$-Sudoku auf ein $4 \times 4$-Sudoku vereinfacht. Eine intuitive Modellierung, die von den Schülerinnen und Schülern selbst erkannt wurde, bestand darin, die Einträge in den Feldern als Variablen $x_{i, j}$ aufzufassen (vgl. [1]). Wobei gilt:

$$
x_{i, j} \in\{1,2,3,4\} \quad i, j \in\{1,2,3,4\}
$$

Die Variablenbelegung $x_{2,3}=4$ bedeutet beispielsweise, dass in der zweiten Zeile in der dritten Spalte eine vier eingetragen ist.

Nach den Regeln des Sudoku müssen nun folgende Nicht-Gleichungen erfüllt sein:

I. In einer Zeile darf nicht zweimal der gleiche Wert eingetragen werden.

$x_{i, 1} \neq x_{i, 2} \quad x_{i, 1} \neq x_{i, 3} \quad x_{i, 1} \neq x_{i, 4}$
$x_{i, 2} \neq x_{i, 3}$
$x_{i, 3} \neq x_{i, 4}$

II. In einer Spalte darf nicht zweimal der gleiche Wert eingetragen sein. 


$$
\begin{aligned}
& x_{1, j} \neq x_{2, j} \quad x_{1, j} \neq x_{3, j} \quad x_{1, j} \neq x_{4, j} \\
& x_{2, j} \neq x_{3, j} \\
& x_{3, j} \neq x_{4, j}
\end{aligned}
$$

III. In einen Block darf nicht zweimal der gleiche Wert eingetragen werden.

$$
\begin{array}{ll}
x_{1,1} \neq x_{2,2} & x_{2,1} \neq x_{1,2} \\
x_{1,3} \neq x_{2,4} & x_{2,3} \neq x_{1,4} \\
x_{3,1} \neq x_{4,2} & x_{4,1} \neq x_{3,2} \\
x_{3,3} \neq x_{4,4} & x_{3,4} \neq x_{4,3}
\end{array}
$$

(Hinweis: Wir können uns hier auf die Diagonalen beschränken, da wir die Ungleichheit in den Reihen und Spalten schon in Regel I und II hergestellt haben.)

Leider ist diese Formulierung durch Nicht-Gleichungen sehr unpraktisch und kaum geeignet, um das Sudoku an ein Lösungsprogramm zu übergeben. In der oben dargestellten $4 \times 4$-Variante haben wir es insgesamt mit 54 solcher Nicht-Gleichungen zu tun. In der üblichen $9 \times 9$-Variante sind hingegen 867 Nicht-Gleichungen zu überprüfen.

Obwohl diese Art und Weise der Darstellung recht unübersichtlich und für die Weiterverarbeitung wenig sinnvoll zu sein scheint, ist es den Schülerinnen und Schülern gelungen, eine mathematische Darstellung von Sudoku zu entwickeln. Das erste Ziel wurde erfüllt: Das Rätsel ist mit seinen Regeln in Mathematik übersetzt worden. Im nächsten Schritt wurde nun nach einer Darstellung gesucht, die mehr den gängigen mathematischen Arbeitsweisen entspricht. Dafür wurde der Fokus für einen Moment weg vom Sudoku auf ein scheinbar völlig anderes Problem gelegt.

Beim sogenannten Damenproblem geht es darum, auf einem Schachbrett acht Damenfiguren so anzuordnen, dass keine Dame eine andere schlagen kann. Bei der mathematischen Darstellung dieses Problems stellten die Schülerinnen und Schüler schnell fest, dass eine sinnvolle Variableneinführung hier eine etwas andere Bedeutung hat. Die 64 Felder des Schachbrettes werden ähnlich wie zuvor beim ersten Sudoku Modell als Variablen $x_{i, j}$ aufgefasst. Es gilt hier:

$$
x_{i, j} \in\{0,1\} \quad i, j \in\{1, \ldots, 8\}
$$

Das besondere an diesem Modell für die Lernenden war die Belegung der Variablen nur mit den Werten o und 1. Der Wert 1 wird angenommen, wenn sich eine Dame auf dem Feld befindet, ansonsten ist der Wert der Variable o. Leicht einsehbar für die Lernenden war, dass in jeder Reihe, in jeder Spalte und in jeder Diagonale die Summe der Variablen höchstens 1 sein darf. Das mathematische Modell für das Damenproblem besteht also aus insgesamt 42 Ungleichungen (je acht Ungleichungen für die Reihen und Spalten und je 13 für die Diagonalen), die alle gleichzeitig erfüllt sein müssen.

Im letzten Schritt der mathematischen Modellbildung wurde nun das Prinzip des mathematischen Modells des Damenproblems auf das Sudoku-Rätsel übertragen. Der entscheidende Schritt war die Einführung von binären Variablen $x_{i, j, k}$ für die Einträge in den Feldern des Sudoku.

$$
x_{i, j, k} \in\{0,1\} \quad i, j, k \in\{1, \ldots, 9\}
$$

Die Variablenbelegung $x_{2,3,8}=1$ bedeutet hier, dass in der zweiten Zeile in der dritten Spalte eine acht eingetragen ist.
Die Belegung $x_{9,7,2}=0$ bedeutet, dass in der neunten Zeile in der siebten Spalte keine zwei eingetragen ist.

An dieser Stelle sei angemerkt, dass die Einführung dieser 0-1-Variablen von den Lernenden durchaus als kognitive Hürde wahrgenommen wurde. Obwohl die Überlegungen zum Damenproblem geholfen haben, das Verständnis zu fördern, fiel es den Schülerinnen und Schülern schwer, sich von der intuitiven Variablenbelegung zu lösen.

Mit Hilfe der genannten Vorüberlegungen konnten nun die ersten drei Regeln des Sudokus als Gleichungen formuliert werden.

I. In jedes Feld wird genau eine Zahl von 1 bis 9 eingetragen.

$$
\sum_{k=1}^{9} x_{i, j, k}=1 \quad \forall i, j
$$

II. In jeder Zeile wird jede Zahl von 1 bis 9 genau einmal eingetragen.

$$
\sum_{i=1}^{9} x_{i, j, k}=1 \quad \forall j, k
$$

III. In jeder Spalte wird jede Zahl von 1 bis 9 genau einmal eingetragen.

$$
\sum_{j=1}^{9} x_{i, j, k}=1 \quad \forall i, k
$$

Die vierte Regel lautet, dass in jedem der neun $3 \times 3$ - Blöcke jede Zahl genau einmal eingetragen wird. Es fiel den Lernenden nicht schwer, für jede der neun Blöcke eine mathematische Darstellung dieser Regel aufzustellen. Für den ersten Block erhält man beispielsweise folgende Gleichungen:

$$
\begin{aligned}
& x_{1,1, k}+x_{1,2, k}+x_{1,3, k}+x_{2,1, k}+x_{2,2, k}+x_{2,3, k} \\
& +x_{3,1, k}+x_{3,2, k}+x_{3,3, k}=1 \quad \forall k
\end{aligned}
$$

Diese Gleichungen für alle Blöcke zusammenfassend aufzuschreiben, überstieg jedoch die Kompetenzen der Schülerinnen und Schüler des 9. Schuljahres. Der Umgang mit symbolischen, formalen und technischen Elementen schien an dieser Stelle noch nicht ausgeprägt genug zu sein. Während die Funktionsweise des Summenzeichens in den ersten beiden Regeln intuitiv nachvollzogen werden konnte, stieBen die Lernenden hier an ihre Grenzen. Die Regel drei wurde statt als Summenformel durch neun einzelne Gleichungen wie oben dargestellt. Der Vollständigkeit halber sei hier die verkürzte Form angegeben.

IV. In jedem Block wird jede Zahl von 1 bis 9 genau einmal eingetragen.

$$
\sum_{l=1}^{3} \sum_{m=1}^{3} x_{3(s-1)+l, 3(t-1)+m, k}=1 \quad \forall s, t \in\{1,2,3\} \quad \forall k
$$

Das entstandene ganzzahlige Programm von Sudoku besteht also aus einem Gleichungssystem mit 729 Variablen und 324 Gleichungen. Zusätzlich werden bei einem klassischen Sudoku-Rätsel mindestens 17 Werte vorgegeben, also 17 Variablen auf 1 fixiert. Um bei einem gegebenen SudokuRätsel zu entscheiden, ob es lösbar ist, kann man das Rätsel als Optimierungsproblem auffassen. Dabei wird die Summe aller Variablen $x_{i, j, k}$ unter den Nebenbedingungen I bis IV und den gegebenen Fixierungen maximiert (oder minimiert). Existiert dieses Maximum (oder Minimum), ist es gleich 81 und das Rätsel ist lösbar. Es handelt sich hierbei also um ein Entscheidungsproblem und kein Optimierungsproblem im klassischen Sinn. 


\subsection{Vom Modell zur Lösung}

Kombinatorische Rätsel wie Sudoku, die in Zeitschriften oder im Internet veröffentlicht werden, sind typischerweise eindeutig lösbar, nicht zuletzt, um Frustration bei den Spielerinnen und Spielern zu verhindern. So war für die Lernenden weniger die Frage nach der Lösbarkeit als viel mehr die konkrete Lösung eines gegebenen Sudokus interessant. Es ging also im nächsten Schritt darum, wie man vom mathematischen Modell zu einer vollständigen Lösung kommt.

Zunächst wurden mithilfe der Modellierungssprache Zimpl [5] verschiedene Sudoku-Rätsel mit gegebenen Startwerten an den Löser SCIP [4] übergeben. Für die Schülerinnen und Schüler war es nun möglich, sogar solche Sudokus, die als extra schwer gekennzeichnet waren, schnell zu lösen.

Aber wie funktioniert so ein Löser? Um Einblicke in die Funktionsweise zu erhalten und dabei elementare Bestandteile von Baumsuchalgorithmen kennen zu lernen, wurde zunächst das Prinzip der Propagierung erläutert. Dazu wurden die Lernenden aufgefordert, eine präzise Beschreibung des intuitiven Vorgehens beim Lösen von Sudokus von Hand anzufertigen. Sie stellten dar, dass mithilfe der bekannten Information und der gegebenen Regeln versucht wird, weitere Information herzuleiten. Wenn zum Beispiel in einem Feld einer Spalte bereits eine Zahl vorgegeben ist, dann ist klar, dass diese Zahl in keinem anderen Feld derselben Spalte stehen darf. Sind in einer Spalte alle bis auf ein Feld mit Zahlen belegt, dann gehört in das verbleibende Feld die verbleibende Zahl.

Das Prinzip der Propagierung wurde also intuitiv angewandt. In diesem Fall entspricht das Vorgehen genau den zwei Propagierungschritten, die man bei Gleichungen der Form „Summe von Binärvariablen gleich 1 “ zur Verfügung hat. Ist eine der Variablen bereits auf 1 fixiert, werden alle anderen Variablen auf o fixiert. Sind alle bis auf eine Variable auf o fixiert, wird die letzte verbleibende Variable auf 1 fixiert. Hat man auf diese Art und Weise mindestens eine weitere Fixierung gefunden, kann man das ganze Verfahren erneut anwenden, bis man keine weiteren Fixierungen mehr findet. Im besten Fall liegt das daran, dass man das Rätsel bereits gelöst hat, also alle Variablen gefunden wurden.

Beispielhaft wurden für eine bestimmte Situation alle nötigen Propagierungsschritte aufgeschrieben. Im folgenden Sudoku wurde der Eintrag in der dritten Zeile und der sechsten Spalte gesucht. Es galt also ein $k \mathrm{zu}$ finden für $x_{3,6, k}=1$.
Den Schülerinnen und Schülern war durch scharfes Hinsehen schnell klar, dass an die gesuchte Position die Zahl 7 gehört. Es ging also vielmehr darum, die Herangehensweise des Solvers zu simulieren und die Funktionsweise der Propagierung sichtbar zu machen.

$$
\begin{aligned}
& \text { 1. } \begin{array}{l}
x_{1,3,7}=1 \Rightarrow x_{1,1,7}+x_{1,2,7}+\sum_{j=4}^{9} x_{1, j, 7}=0 \quad \Rightarrow x_{1,4,7}= \\
x_{1,5,7}=x_{1,6,7}=0
\end{array} \\
& \text { 2. } \begin{array}{l}
x_{2,8,7}=1 \Rightarrow \sum_{j=1}^{7} x_{2, j, 7}+x_{2,9,7}=0 \Rightarrow x_{2,4,7}=x_{2,5,7}= \\
x_{2,6,7}=0
\end{array} \\
& \text { 3. } x_{7,4,7}=1 \Rightarrow \sum_{i=1}^{6} x_{i, 4,7}+x_{8,4,7}+x_{9,4,7}=0 \Rightarrow x_{3,4,7}=0 \\
& \text { 4. } x_{3,5,8}=1 \Rightarrow \sum_{k=1}^{7} x_{3,5, k}+x_{3,5,9}=0 \Rightarrow x_{3,5,7}=0 \\
& \text { 5. } \begin{array}{l}
\left(x_{1,4,7}=x_{1,5,7}=x_{1,6,7}=x_{2,4,7}=x_{2,5,7}=x_{2,6,7}=x_{3,4,7}=\right. \\
\left.x_{3,5,7}=0\right) \Rightarrow x_{3,6,7}=1
\end{array}
\end{aligned}
$$

Gebräuchliche Löser von ganzzahligen Programmen wie SCIP lösen alle Sudoku-Rätsel, ohne über Propagierungsverfahren hinaus zu gehen. Nichtsdestotrotz stellte sich für die Lernenden an dieser Stelle die Frage, was passiert, wenn man mit dem reinen Abarbeiten von logischen Schlussfolgerungen nicht mehr weiter kommt. Dieser Ansatz wurde genutzt, um das Prinzip des Probing einzuführen. Als Hinführung wurde folgende Situation betrachtet, die eine mögliche Belegung für die obere rechte Ecke eines SudokuRätsels zeigt.

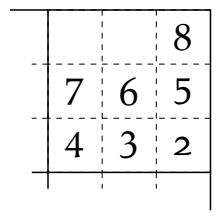

Offenbar gilt hier $x_{1,8,1}=1$ oder $x_{1,8,9}=1$. Im Zuge des Probings fixiert man jetzt eine der beiden Variablen vorübergehend auf 1 und führt mit dieser Fixierung Propagierung durch. Alle sich ergebenden Variablenfixierungen merkt man sich. Dann fixiert man die andere Variable vorübergehend auf 1, wendet wieder Propagierung an und merkt sich das Ergebnis. Nun vergleicht man. Jetzt kann passieren, dass eine andere Variable in beiden Fällen jeweils auf 1 fixiert wurde, also unabhängig von der Belegung der beiden obigen Variablen diesen Wert annimmt. Es wurde also eine weitere sichere Fixierung gefunden, mit der weiter Propagierung durchgeführt werden kann.

Es kann auch passieren, dass eine der beiden oben durchgeführten Belegungen zu einem Widerspruch führen. Hier könnte beispielsweise aus der Annahme $x_{1,8,1}=1$ folgen, dass $x_{4,7,4}=1$ gelten muss. Dies stellt einen Widerspruch zu den bisherigen Fixierungen dar, also muss $x_{1,8,1}=0$ und so $x_{1,8,9}=1$ gelten.

Obwohl das Thema Probing nur in konstruierten Situationen wie oben mit den Lernenden besprochen werden konnte, bekamen die Schülerinnen und Schüler fundierte Einblicke in die Funktionsweise. Darauf aufbauend wurde mit den Lernenden das Konzept des Branching thematisiert, was allerdings ein wenig weg vom eigentliche SudokuRätsel führte und an anderer Stelle detaillierter vorgestellt wird. 
Im Zuge des vorgestellten Unterrichtsvorhabens sind die Schülerinnen und Schüler mit aktuellen Prinzipien eines wichtigen Teils der angewandten Mathematik in Berührung gekommen. Sie haben einen Teil der Mathematik kennen gelernt, der im schulischen Alltag kaum thematisiert wird und konnten den Blick ein wenig über den Tellerrand der Schulmathematik werfen. Die authentische Auseinandersetzung mit wichtigen Werkzeugen dieses Teils der Mathematik wurde unterstützt durch einen Besuch des Zuse Instituts Berlin. Den Lernenden wurden durch Vorträge und Führungen weitere Perspektiven auf das Fach und das Berufs- und Forschungsfeld Mathematik eröffnet.

Im Sinne des kompetenzorientierten Mathematikunterrichts wurden im besonderen Maße die Modellierungsfähigkeiten der Lernenden gefördert. Die Auseinandersetzung mit dem Lerngegenstand erfolgte in enger Begleitung lehrkraftunterstützt. Anschließend folgte auf Grundlage der erarbeiteten Wissensbasis eine Phase der selbstständigen Forschung. Dabei bearbeiteten die Schülerinnen und Schüler selbst gestellte Fragen wie „Nach welchen Kriterien wird ein Sudoku als ,schwer' oder ,einfach' bezeichnet?" oder „Welche ,Spezialregeln' sind denkbar und welchen Einfluss haben diese Regeln darauf, ob das Sudoku eindeutig lösbar ist, wie viele Zahlen mindestens dazu angegeben werden müssen und wie viele verschiedene Sudokus es gibt" (vgl. [2]). Diese Phase wies einen hohen Grad an Selbstdifferen- zierung auf. Die Lernenden arbeiteten gemäß ihren Fähigkeiten und wählten selbst den Komplexitätsgrad der Forschung. Durch den fachinhaltlichen Fokus der Einheit und die Beschäftigung mit einem aus der Schule unbekannten Teilgebiet der Mathematik, das aktuell von herausragender wissenschaftlicher Bedeutung ist, erfuhren die Lernenden ein sehr authentisches Forschungserlebnis. Durch den forschenden Ansatz fand ein produktiver Umgang mit dem Lerngegenstand statt, der zu einem tiefen Verständnis für die Prinzipien der mathematischen Optimierung führte. Die Mathematik sowie die Arbeitsweisen wurden sichtbar gemacht und systematisiert.

\section{Literatur}

[1] Kaibel, V., Koch, T.: Mathematik für den Volkssport. In: Mitteilungen der DMV 14, 93-96 (2006).

[2] Lutz-Westphal, B., Schulte, A.: Mathematische Forschung - Was forschendes Lernen im Mathematikunterricht aus der Praxis lernen kann. In: Beiträge zum Mathematikunterricht 2016. WTMVerlag, Münster (2016).

[3] Landesinstitut für Schule und Medien Berlin-Brandenburg (Hg.): Rahmenlehrplan Mathematik (2016). bildungsserver. berlin-brandenburg.de/rlp-online/c-faecher/mathematik/ kompetenzentwicklung

[4] SCIP - Solving Constraint Integer Programs. http://scip.zib.de

[5] ZIMPL - Zuse Institut Mathematical Programming Language. http://zimpl.zib.de

Alexander Schulte, Freie Universität Berlin, Institut für Mathematik,

Didaktik der Mathematik, Arnimallee 3, 14195 Berlin

alexander.schulte@fu-berlin.de

Alexander Schulte ist wissenschaftlicher Mitarbeiter in der AG Didaktik der Mathematik der Freien Universität Berlin. Sein Promotionsvorhaben beschäftigt sich mit dem Konzept des „Forschenden Lernens" und dabei schwerpunktmäßig mit der Analyse und Sichtbarmachung mathematischer Forschungsprozesse in der wissenschaftlichen Praxis. Außerdem ist er mit den Fächern Mathematik und Geschichte als Lehrer am Berliner Heinz-Berggruen-Gymnasium tätig. 